\title{
TITLE: Meta-analysis of the analgesic efficacy of clonidine for postop pain
}

AUTHORS: Alan J. Chaput, PharmD, MD, MSc, W. Scott Beattie, MD, PhD

AFFILIATION: Department of Anaesthesia, University Health Network - TGH, Eaton

North 3-462, 200 Elizabeth Street, Toronto, ON, M5G 2C4

INTRODUCTION: Regional anaesthesia using both local anesthetics (LA) and narcotics are often used for postoperative pain management. Alpha-2 adrenergic agonists have been demonstrated to be effective at reducing narcotic and LA requirements and improving pain outcomes. The objectives of this systematic review are to assess the analgesic efficacy of clonidine when used as an adjunct to epidurally administered LA for the management of acute postoperative pain and to assess adverse events.

METHODS: MEDLINE, EMBASE, and Cochrane CENTRAL register searched from inception for RCT's in adult patients administered therapeutic doses of LA epidurally and either clonidine or placebo. Reference lists of review articles and included studies were reviewed for additional citations. Studies had to report on at least one of: VAS, time to first analgesic, or analgesic consumption postoperatively. Information on patients, methods, interventions, outcomes and adverse events were collected in duplicate. Methodologic quality was assessed using an instrument designed by the Cochrane Anaesthesia Review Group. Primary meta-analyses were conducted using a random effects model. Continuous data summarized as weighted mean difference (WMD) with $95 \% \mathrm{CI}$ and dichotomous data summarized as odds ratio (OR) with $95 \%$ CI. Sensitivity analysis conducted using fixed effects models and excluding abstracts.

RESULTS: The search yielded 210 articles. Eleven articles met the inclusion and exclusion criteria. Median methodologic quality score was 7 out of a possible 10 . Clonidine was administered epidurally in all studies and was associated with significantly decreased VAS scores at 1,2,8,12 and 48 hours postoperatively, a trend to decreased VAS scores was noted at all other time points, decreased morphine IV consumption (28.9 $\mathrm{mg}$ in $1^{\text {st }} 24$ hours), increased time to analgesic use and increased odds of sedation with OR 5.89 (95\% CI: $2.3,15.2)$ and bradycardia with OR 5.89 (95\% CI: 2.2, 15.8). No increase in odds of hypotension was seen.

DISCUSSION: Clonidine administration perioperatively improves pain outcomes and limits narcotic exposure with little risk of adverse events and is a useful adjunct to epidurally administered LA. Further studies are required to determine if oral clonidine has similar effects on pain outcomes and adverse events in this population. 